\title{
The Time of Prenatal Immune Challenge Determines the Specificity of Inflammation-Mediated Brain and Behavioral Pathology
}

\author{
Urs Meyer, ${ }^{1 *}$ Myriel Nyffeler, ${ }^{1 *}$ Andrea Engler, ${ }^{2}$ Adrian Urwyler, ${ }^{2}$ Manfred Schedlowski, ${ }^{2}$ Irene Knuesel, ${ }^{1}$ \\ Benjamin K. Yee, ${ }^{1}$ and Joram Feldon ${ }^{1}$ \\ ${ }^{1}$ Laboratory of Behavioural Neurobiology, Swiss Federal Institute of Technology Zurich, CH-8603 Schwerzenbach, Switzerland, and ${ }^{2}$ Laboratory of \\ Psychology and Behavioural Immunobiology, Swiss Federal Institute of Technology Zurich, CH-8092 Zurich, Switzerland
}

\begin{abstract}
Disturbance to early brain development is implicated in several neuropsychiatric disorders including autism, schizophrenia, and mental retardation. Epidemiological studies have indicated that the risk of developing these disorders is enhanced by prenatal maternal infection, presumably as a result of neurodevelopmental defects triggered by cytokine-related inflammatory events. Here, we demonstrate that the effects of maternal immune challenge between middle and late gestation periods in mice are dissociable in terms of fetal brain cytokine responses to maternal inflammation and the pathological consequences in brain and behavior. Specifically, the relative expression of pro- and anti-inflammatory cytokines in the fetal brains in response to maternal immune challenge may be an important determinant among other developmental factors for the precise pathological profile emerging in later life. Thus, the middle and late gestation periods correspond to two windows with differing vulnerability to adult behavioral dysfunction, brain neuropathology in early adolescence, and of the acute cytokine responses in the fetal brain.
\end{abstract}

Key words: apoptosis; cytokines; inflammation; neurogenesis; prenatal; Reelin

\section{Introduction}

Maternal bacterial and viral infections during pregnancy represent a risk factor in several neuropsychiatric disorders with a presumed neurodevelopmental origin, including schizophrenia (Mednick et al., 1988; O’Callaghan et al., 1994; Brown et al., 2004b), autism (Rodier and Hyman, 1998; Miller et al., 2005), and mental retardation (Rantakallio and von Wendt, 1985; Revello and Gerna, 2004). Although the mechanisms underlying this epidemiological relationship remain unclear, the maternal cytokine-associated inflammatory response to infection may be the crucial link, because the identity of the pathogen seems irrelevant (Gilmore and Jarskog, 1997; Buka et al., 2001; Pearce, 2001; Brown et al., 2004a). Similar inflammatory mechanisms have also been suggested to underlie the association of chorioamnionitis and the development of cerebral palsy (Dammann and Leviton, 1997, 2000; Wu and Colford, 2000; Wu, 2002). Animal experimentation has also confirmed that, in the absence of a pathogenic agent, cytokine-releasing treatment to pregnant mothers is suffi-

Received Jan. 10, 2006; revised March 16, 2006; accepted March 18, 2006

This work was supported by the Swiss Federal Institute of Technology Zurich, with additional support from the National Centre for Competence in Research: Neural Plasticity and Repair, Swiss National Science Foundation. We are extremely grateful to Peter Schmid for his technical assistance. We also remain indebted to Jeanne Michel and Pascal Guela for their care of the animals and to Dr. Frank Bootz for his veterinary expertise.

*U.M. and M.N. contributed equally to this work.

Correspondence should be addressed to Dr. Benjamin K. Yee, Laboratory of Behavioural Neurobiology, Swiss Federal Institute of Technology Zurich, Schorenstrasse 16, CH-8603 Schwerzenbach, Switzerland. E-mail: byee@ethz.ch.

DOI:10.1523/JNEUROSCI.0099-06.2006

Copyright $\odot 2006$ Society for Neuroscience $\quad$ 0270-6474/06/264752-12\$15.00/0 cient to induce multiple psychopathology in the offspring later in adulthood (Shi et al., 2003; Zuckerman et al., 2003; Meyer et al., 2005, 2006).

In addition to their immunological roles (Curfs et al., 1997), cytokines can modulate neuronal survival (Marx et al., 2001) and differentiation (Ling et al., 1998; Potter et al., 1999), as well as dendrite growth and complexity (Gilmore et al., 2004), thereby exerting multiple effects on the developing CNS. Intense elevation of inflammatory cytokine levels in the fetal environment may therefore adversely affect neurodevelopment and contribute to disease processes implicated in schizophrenia, autism, and mental retardation. However, direct evidence for this causal link is lacking, and the mechanisms whereby infection-induced elevation of cytokine levels during fetal life can interfere with neurodevelopmental events remain poorly understood.

The functioning of the maternal host's immune system exhibits considerable fluctuations as pregnancy progresses (Sargent, 1993). The associated changes in cytokine responses to an immunological challenge may therefore critically modulate the specificity of inflammatory events in the developing fetus and thus allow the dissection of the relative potentials of different species of cytokines to precipitate brain mal-development and related aberrations in behavior and cognition emerging in later life. At the same time, this suggests that the developing nervous system may not be uniformly vulnerable to maternal infection throughout gestation. Indeed, epidemiological data suggest that maternal infection during early-to-mid human pregnancy is more likely to be associated with long-term developmental brain and behav- 
a

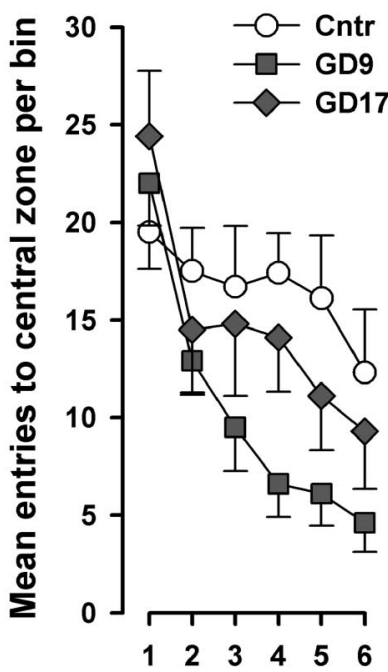

10-min bins

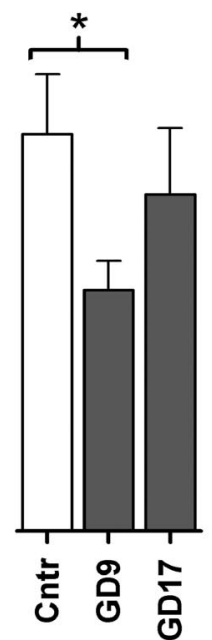

C
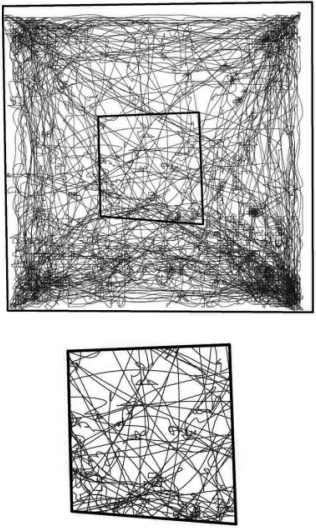

Cntr
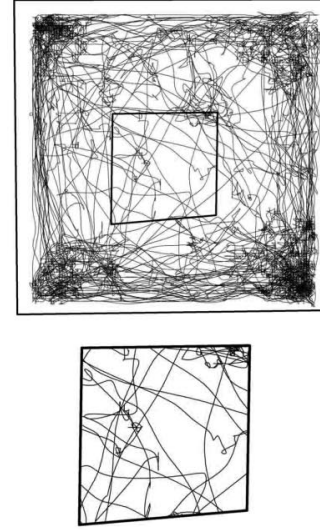

GD9 b

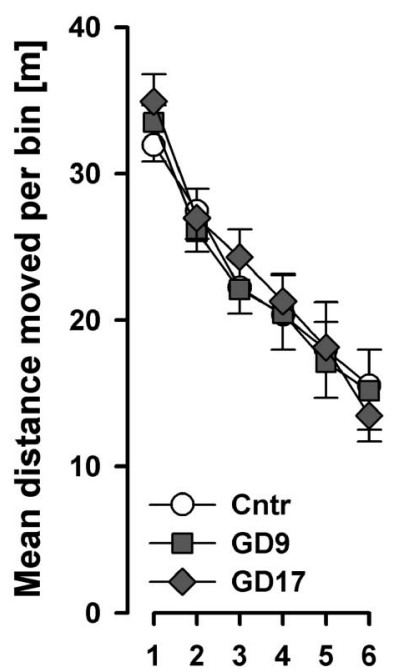

10-min bins
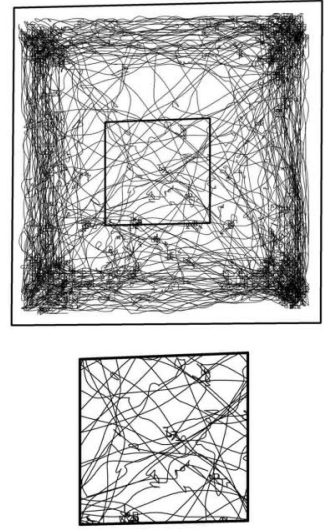

GD17
Figure 1. Middle but not late prenatal immune challenge suppresses explorative behavior in adulthood. $\boldsymbol{a}$, Adult mice born to Polyl:C-exposed mothers on GD9 showed a significant deficit in exploratory behavior compared with control offspring, as evident by the reduction in the frequency to enter the central area in the open field. This effect was not observed in animals born to Polyl:C-treated mothers on GD17. ${ }^{*} p<0.05$, statistical significance based on Fisher's LSD post hoc comparison. $\boldsymbol{b}$, No group difference in basal locomotor activity was revealed, therefore the suppression of spatial exploration in GD9-Polyl:C offspring cannot be attributed to any changes in general locomotor activity. $\boldsymbol{c}$, The three panels illustrate the representative pattern of spatial exploration of the three groups in the entire open field (top row) and in the central area (bottom row). $n=10$ ( 5 males and 5 females) in each treatment group. All values are mean \pm SEM. (ntr, Control.

ioral abnormality in the offspring (Mednick et al., 1988; Rodier and Hyman, 1998; Brown et al., 2004b).

Here, an intense but time-limited inflammatory cytokine response in pregnant mouse dams was induced by an acute systemic challenge of the viral mimic polyriboinosinicpolyribocytidilic acid (PolyI:C), a synthetic analog of doublestranded RNA (Fortier et al., 2004). It was conducted in pregnant mouse dams on gestation day 9 (GD9) or GD17 at a proven functionally effective dose (Meyer et al., 2005, 2006). The two gestation days selected here correspond to the first-to-second and second-to-third trimesters of human pregnancy, respectively, with respect to developmental biology and percentage of gestation from mice to human (Kaufman, 2003).

\section{Materials and Methods \\ Animals}

Female and male C57BL6/J breeders were obtained from our in-house specific pathogenfree colony at the age of 10-14 weeks. Littermates of the same sex were kept in groups of three to five mice. Breeding began after 2 weeks of acclimatization to the new animal holding room. The breeding procedure and the verification of pregnancy have been fully described previously (Meyer et al., 2005). All procedures described in the present study had been approved previously by the Zurich Cantonal Veterinary Office and are in agreement with the Principles of Laboratory Animal Care (National Institutes of Health publication number 86-23, revised 1985).

\section{Prenatal treatment}

Pregnant dams on GD9 or GD17 received either a single injection of PolyI:C or vehicle solution via the intravenous route at the tail vein under mild physical constraint. PolyI:C (potassium salt) was obtained from Sigma-Aldrich (Buchs, St. Gallen, Switzerland) and dissolved in saline to obtain the desired dosage $(5 \mathrm{mg} / \mathrm{kg}$, calculated based on the pure form PolyI:C). The volume of injection was $5 \mathrm{ml} / \mathrm{kg}$. All animals were immediately returned to the home cage after the injection procedure. A group of pregnant dams were left untreated throughout pregnancy, which served as an additional control group for the behavioral experiments.

For the purpose of assessing the acute effects of PolyI:C administration on the cytokine protein levels in the maternal serum and fetal brain tissue, as well as on the relative gene expression levels in the fetal brain, PolyI:C- or vehicletreated pregnant dams were killed by decapitation 3 or $6 \mathrm{~h}$ after treatment. Trunk blood was collected into Eppendorf (Hamburg, Germany) tubes, and fetal brains were extracted under visual guidance using a dissecting microscope (Stemi 2000-C; Zeiss, Oberkochen, Germany) as described previously (Meyer et al., 2005). Trunk blood was allowed to clot at room temperature for $1 \mathrm{~h}$ before centrifugation at 12,000 $\mathrm{rpm}$ for $4 \mathrm{~min}$ at $4^{\circ} \mathrm{C}$. The resulting serum from each animal was subdivided to permit storage $\left(-80^{\circ} \mathrm{C}\right)$ until the cytokine assays were performed. The brain samples collected for the cytokine protein assay were stored at $-80^{\circ} \mathrm{C}$ until further processing. Fetal brain samples assigned to the gene expression assays were processed immediately after tissue collection (see below).

Cytokine protein assays

Fetal brain samples were placed in 10 vol of Tris- $\mathrm{HCl}$ buffer $(50 \mathrm{~mm}, \mathrm{pH}$ 7.4) with $\mathrm{NaCl}(0.6 \mathrm{M})$, Triton X-100 (0.2\%), and BSA (0.5\%) containing freshly dissolved protease inhibitors: benzamidine $(1 \mathrm{mM})$, benzethonium chloride $(0.1 \mathrm{~mm})$, and phenylmethylsulfonyl fluoride $(0.1 \mathrm{~mm})$. Samples were homogenized (TissueTearor; BioSpec Products, Bartlesville, OK) for $10 \mathrm{~s}$, sonicated (Vibra Cell; Sonics \& Materials, Newtown, CT) for $20 \mathrm{~s}$ at $10 \mathrm{mV}$, and centrifuged at $12,000 \mathrm{rpm}$ for $20 \mathrm{~min}$ at $4^{\circ} \mathrm{C}$. The supernatants were aliquoted and frozen at $-80^{\circ} \mathrm{C}$ until the cytokine assays were performed.

Cytokine protein levels in the maternal serum and fetal brain tissue were measured using a multiplexed particle-based flow cytometric cytokine assay (Vignali, 2000). Fluorokine MAP (Multi Analyte Profiling) 
mouse kits for interleukin $1 \beta$ (IL- $1 \beta$ ), IL-6, IL-10, and tumor necrosis factor $\alpha$ (TNF- $\alpha$ ) were purchased from R \& D Systems (WiesbadenNordenstadt, Germany). The procedures closely followed the manufacturer's instructions. The analysis was conducted using a conventional flow cytometer (Cyan ADP; DakoCytomation, Glostrup, Denmark). The detection limits were $2.7 \mathrm{pg} / \mathrm{ml}$ for IL- $1 \beta, 0.1 \mathrm{pg} / \mathrm{ml}$ for IL-6, $0.5 \mathrm{pg} / \mathrm{ml}$ for IL-10, and $0.4 \mathrm{pg} / \mathrm{ml}$ for TNF- $\alpha$.

\section{Cytokine gene expression assays}

Fetal brain samples were placed into Trizol (Invitrogen, Basel, Switzerland), homogenized with a TissueTearor (BioSpec Products), and stored at $-80^{\circ} \mathrm{C}$. Total RNA was isolated later according to the manufacturer's instructions. The RNA was DNase I treated and further purified on RNeasy Micro kit spin columns (Qiagen, Hombrechtikon, Switzerland). Total RNA was reverse transcribed with Moloney murine leukemia virus reverse transcriptase (Invitrogen) and random hexamer primers (Invitrogen). Gene expression analysis using Taqman gene expression assays (Applied Biosystems, Rotkreuz, Switzerland) for IL-1 $\beta$ (Mm00434228_m1), IL-6 (Mm00446190_m1), IL-10 (Mm439616_m1), TNF- $\alpha$ (Mm00443258_m1), and 18S rRNA (\#4319413E) was performed on an Applied Biosystems (Foster City, CA) 7500 Real-Time PCR. Relative gene expression was calculated according to the $2^{--\Delta \Delta \mathrm{Cr}}$ method (Livak and Schmittgen, 2001).

\section{Immunohistochemistry}

At the age of $24 \mathrm{~d}$, eight PolyI:C-exposed (four per sex) and four control (saline injected, two per sex) mice were anesthetized with an overdose of sodium pentobarbital $(5 \mathrm{mg} / \mathrm{kg})$ and perfused transcardially with $4 \%$ phosphate-buffered paraformaldehyde solution with picric acid. They were postfixed overnight and cryoprotected in 30\% sucrose solution for $48 \mathrm{~h}$ at $4^{\circ} \mathrm{C}$. Sections ( $30 \mu \mathrm{m}$ thick) were cut coronally from frozen blocks with a sliding microtome, and five serial sections from bregma 2.20 to $-3.80 \mathrm{~mm}$ were collected, rinsed in PBS, and stored at $-20^{\circ} \mathrm{C}$ in antifreeze until further processing.

For immunostaining, the slices were rinsed three times for $10 \mathrm{~min}$ in PBS. Blocking was done in PBS, $0.3 \%$ Triton X-100, and 5\% normal serum for $1 \mathrm{~h}$ at room temperature. The following primary antibodies were used: horse anti-doublecortin (DCX) (1:1000; Santa Cruz Biotechnology, Santa Cruz, CA), rabbit anti-cleaved caspase-3 (1:600; Cell Signaling Technology, Beverly, MA), rabbit anti-GFAP (1:5000; DakoCytomation), and mouse anti-Reelin (1:700; Chemicon, Temecula CA). They were diluted in PBS containing $0.3 \%$ Triton X-100 and $2 \%$ normal serum and incubated overnight at room temperature. After three washes with PBS (10 min each), the sections were incubated for $1 \mathrm{~h}$ with the biotinylated secondary antibodies diluted 1:500 in PBS containing 2\% normal serum and $0.3 \%$ Triton $\mathrm{X}-100$. Sections were washed again three times for $10 \mathrm{~min}$ in PBS and incubated with the Vectastain kit (Vector Laboratories, Burlingame, CA) diluted in PBS for $1 \mathrm{~h}$. After three rinses in $0.1 \mathrm{M}$ Tris- $\mathrm{HCl}, \mathrm{pH} 7.4$, the sections were stained with $1.25 \% 3,3-$ diaminobenzidine and $0.08 \% \mathrm{H}_{2} \mathrm{O}_{2}$ for $8-20 \mathrm{~min}$, rinsed again four times in PBS, dehydrated, and coverslipped with Eukitt (Kindler, Freiburg, Germany). The GFAP-labeled slices were counterstained with $0.25 \%$ cresyl violet according to standard protocols. After staining, the sections were dehydrated through an alcohol series, cleared with xylene, and coverslipped with Eukitt.

\section{Densitometry}

All analyses were performed blind to the treatment and gender classification of the animals included in the study. Fifteen coronal brain sections per animal were analyzed with a Zeiss Axioplan microscope, and digital images of the DCX stainings were acquired using a digital camera (Axiocam; Zeiss). Exposure times were set so that pixel brightness was never saturated and were held constant during acquisition of all images for each experiment. Densitometry measurements of the DCX immunoreactivity were performed using ImageJ software (National Institutes of Health, Bethesda, MD). Pixel brightness was measured in the subgranular and outer granular cell layer of both blades of the dentate gyrus and the corpus callosum (serving as background area) by placing a rectangular box of $100 \times 100$ pixels in the respective area. The background-corrected optical densities obtained from both brain hemispheres were averaged per animal.

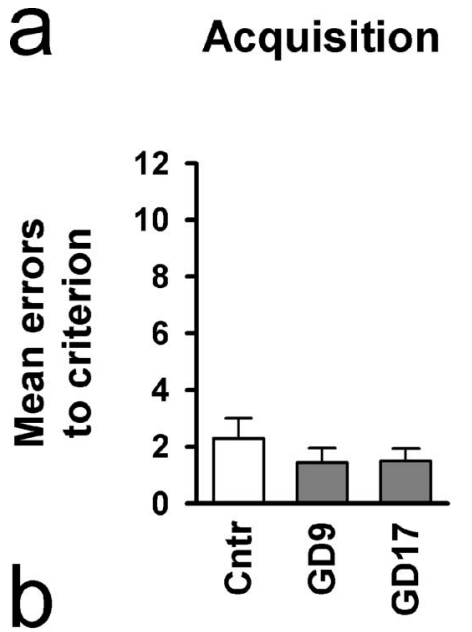

\section{Reversal}

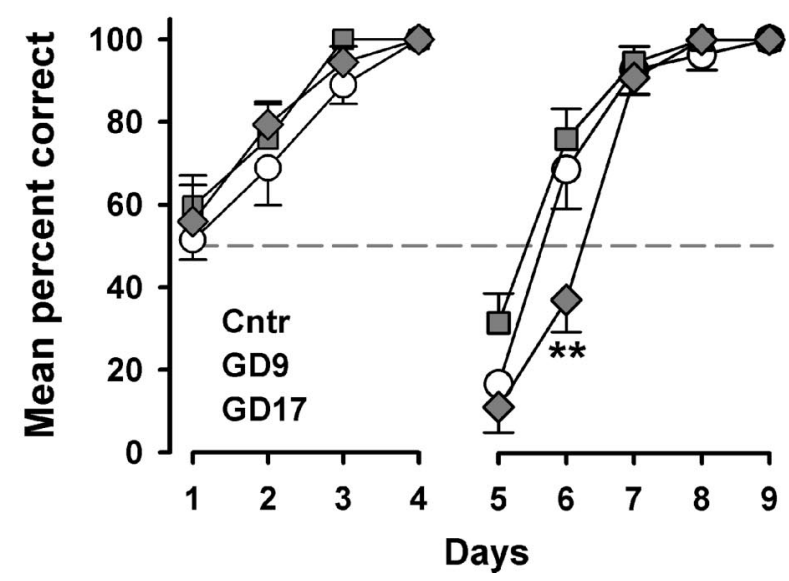

Figure 2. Emergence of perseverative behavior after late but not mid pregnancy immune challenge. Neither GD9-Polyl:C nor GD17-Polyl:C treatment affected the acquisition of a leftright discrimination task, as evident by both the measures of error to criterion $(\boldsymbol{a})$ and percentage correct $(\boldsymbol{b})$ on successive training days. A clear selective deficit in the GD17-Polyl:C group emerged in the subsequent reversal training, indicating a perseverative phenotype. A reversal effect was evident in all groups as suggested by the below-chance performance (represented by the dashed line) on the first day of reversal learning $(\boldsymbol{b})$. The reversal effectpersisted into the second day of reversal training in the GD17-Polyl:C group but not the other treatment conditions. $n=9$ (males) in each treatment group. All values are mean \pm SEM. ${ }^{*} p<0.05,{ }^{* *} p<0.01$, and ${ }^{* * *} p<0.001$, statistical significance based on Fisher's LSD post hoc analysis. Cntr, Control.

\section{Stereological estimation of Reelin-positive neuron}

The total number of Reelin-positive neurons in the CA1 stratum oriens and dentate gyrus molecular layer of both hemispheres was determined using the optical fractionator method (Gundersen et al., 1988). With the aid of the image analysis computer software Stereo Investigator (version 6.50.1; Microbrightfield, Colchester, VT), every section of a one-in-five series was measured, resulting in an average of $12-15$ sections per animal. The following sampling parameters were used: (1) a fixed counting frame with a width of $50 \mu \mathrm{m}$ and a length of $50 \mu \mathrm{m}$; and (2) a sampling grid size of $250 \times 250 \mu \mathrm{m}$. The counting frames were placed randomly at the intersections of the grid within the outlined structure of interest by the software. The cells were counted following the unbiased sampling rule (Howard and Reed, 2005) using the $40 \times$ oil lens [numerical aperture (NA), 1.3] and included in the measurement when they came into focus within the optical dissector (height, $10 \mu \mathrm{m}$ ). For the analysis, total estimated Reelin-positive neurons were taken into account for each animal.

\section{Counting of caspase-3-positive neurons}

Cleaved caspase-3-positive cells in the dentate gyrus were counted exhaustively on both hemispheres of seven to nine stained sections per 

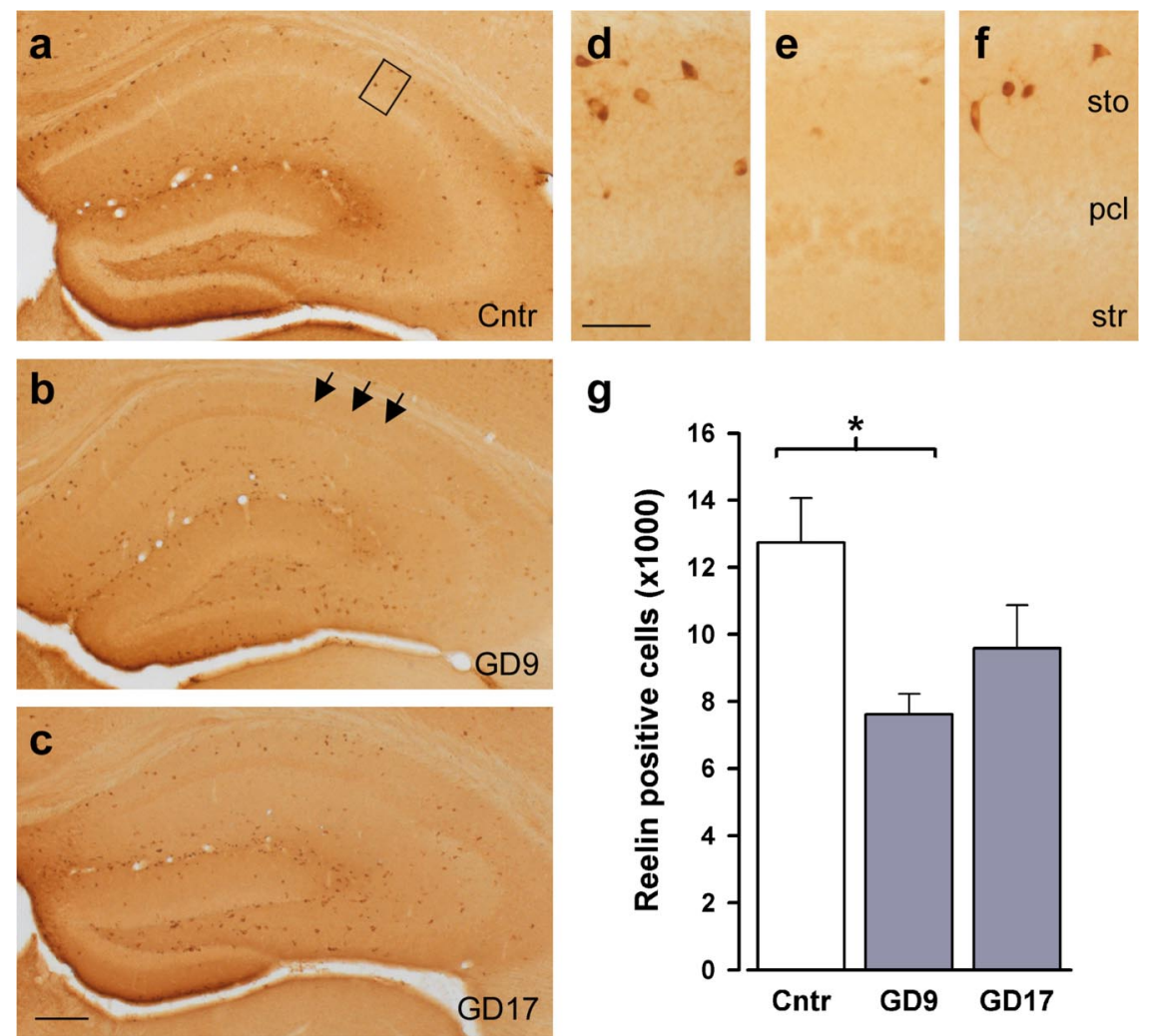

Figure 3. Enhanced sensitivity to inflammation-mediated disturbances in postnatal Reelin expression in the brains of offspring after mid-pregnancy immune challenge. $\boldsymbol{a}-\boldsymbol{c}$, Distribution of Reelin-immunoreactive cells in the hippocampal formation and dentate gyrus of representative control animals (a) and animals subjected to prenatal Polyl:C exposure on GD9 (b) or GD17 (c). As evident in the images at a higher magnification (indicated by the square in $\boldsymbol{a}$ ), prenatal Polyl:( exposure affected Reelin expression particularly in the stratum oriens (sto) of the CA1 subfield. The arrows in $\boldsymbol{b}$ indicate the lack of Reelin-positive cells in the entire stratum oriens of GD9-Polyl:C offspring. $\boldsymbol{e}, \boldsymbol{g}$, This effect was particularly pronounced GD9-Polyl:C-exposed animals $(\boldsymbol{e})$ relative to controls $(\boldsymbol{d})$, leading to a significant reduction in Reelin-positive cells in the brains of GD9-Polyl:C offspring compared with controls (g).f, Prenatal Polyl:C exposure on GD17 only resulted in a marginal reduction in postnatal Reelin immunoreactivity. $n=4$ in each treatment group. The values in $\boldsymbol{g}$ are mean \pm SEM. ${ }^{*} p<0.05$, statistical significance based on Fisher's LSD post hoc analysis. Scale bars: $\boldsymbol{a}-\boldsymbol{c}, 500 \mu \mathrm{m} ; \boldsymbol{d}, \boldsymbol{e}, 50 \mu \mathrm{m}$. Cntr, Control; sto, stratum oriens; str, stratum radiatum; pcl, pyramidal cell layer.
$45 \mathrm{~cm}$ long. They joined at the center of the water tank. A piece of cylindrical clear Plexiglas, $7 \mathrm{~cm}$ in diameter and $18.5 \mathrm{~cm}$ high, was positioned at the end of either the left or right goal arm and served as the escape platform.

In acquisition training, the animals were required to learn to discriminate the left and right goal arms, with only one of them leading to an escape platform hidden at the far end. For onehalf of the animals in each group, the left arm was correct, and for the others, the right arm was correct. The platform location remained unchanged until the end of acquisition. There were six trials per daily session, conducted at an intertrial interval of $10 \mathrm{~min}$. To begin a trial, the animals were placed at the beginning of the start arm and allowed up to $1 \mathrm{~min}$ to choose between the two goal arms. Once its entire body had entered into an arm, a guillotine door was lowered to prevent the animal from retracing. If the animal chose the correct arm, it was allowed to remain on the platform for $10 \mathrm{~s}$, after which it was removed from the maze and returned to a holding cage. When the incorrect arm was chosen, the animal was confined to the arm for $\sim 10$ $s$ and then removed from the maze to a holding cage. Acquisition training continued until an animal had reached criterion performance of 11 correct responses across 2 consecutive days (i.e., of 12 trials). Afterward, the platform location was moved to the other, previously incorrect, arm to assess reversal learning. Reversal training continued until an animal reached criterion performance once again. The percentage of correct arm choices as well as errors to criterion performance were recorded for each animal during acquisition and reversal training. Only male offspring were tested in the discrimination reversal learning paradigm, because of excessive floating behavior in the female mice.

\section{Statistical analysis}

animal using live microscopy with a $10 \times$ objective (NA, 0.45). Cell counts were averaged per hemisphere and animal.

\section{Behavioral assays}

The offspring were weaned and sexed at postnatal day 24 (P24). The pups were weighed, and littermates of the same sex were caged separately and maintained as described previously (Meyer et al., 2005). Behavioral testing commenced when they reached 14-16 weeks of age. Each prenatal treatment group consisted of subjects derived from multiple independent litters (at least five in each treatment condition).

Open-field exploration. The apparatus comprised four identical $40 \times$ $40 \mathrm{~cm}^{2}$ rectangular white arenas. Data collection was performed using the Ethovision tracking system (Noldus Information Technology, Wageningen, The Netherlands) as described previously (Meyer et al., 2005). To begin, the animals were gently placed in the center of the arena and allowed to explore for $1 \mathrm{~h}$. For the purpose of data collection, the arena was conceptually partitioned into two zones: a center zone (measuring $13.5 \times 13.5 \mathrm{~cm}^{2}$ ) in the middle of the arena and a peripheral zone occupying the remaining area. Offspring of both sexes were included in the open-field exploration test.

Discrimination reversal learning. The apparatus consisted of a modified white circular tank measuring $1 \mathrm{~m}$ in diameter. It was filled daily with fresh water to a depth of $19 \mathrm{~cm}$, with the temperature of the water maintained at $\sim 24^{\circ} \mathrm{C}$. Opaque Plexiglas partitions were positioned vertically into the water to form three interconnected corridors (10 cm wide) in the shape of a "T." The partition walls extended to a height of $18 \mathrm{~cm}$ above the water surface. The vertical start arm was $30 \mathrm{~cm}$ long, and the two parallel choice arms were
Behavioral and immunohistochemical as well as maternal serum cytokine data were analyzed using parametric ANOVA, followed by Fisher's LSD post hoc comparisons whenever a main effect or interaction attained statistical significance. Fetal brain cytokine protein and gene expression data were analyzed using independent Student's $t$ tests (two-tailed). Statistical significance was set at $p<0.05$. Analyses were conducted using the statistical software StatView (version 5.0) implemented on a personal computer running the Windows XP operating system. Equal numbers of offspring born to vehicle-treated and nontreated dams were combined into a single control group in the final analysis and in the presentation of the behavioral results after confirmation that they did not differ from each other in terms of these measures (see also Meyer et al., 2005, 2006). The factor sex was also dropped to enhance statistical power in all the immunohistochemical analyses because it never attained significance.

\section{Results}

\section{Dissociating the adult psychopathology after prenatal} immune activation at middle and late pregnancy

The effects of prenatal immune challenge on behavior are expected to achieve their prominence when the affected individuals reach adult age (Zuckerman et al., 2003). Here, we show that GD9 but not GD17 prenatal immune activation reduced open-field exploration (Fig. 1), whereas GD17 but not GD9 immune activation led to perseverative behavior (Fig. 2). 
First, we analyzed the spatial exploration in an open field by focusing on the number of entries the animals ventured into the central area of the field during a $1 \mathrm{~h}$ test period. Animals in the GD9-PolyI:C group showed a marked reduction in this measure that is most obvious in the second half of the test. The effect of GD17-PolyI:C treatment on this measure was weak by comparison and statistically nonsignificant (Fig. $1 a, c)$. A $2 \times 3 \times 6$ (sex $\times$ treatment $\times 10$ min bins) split-plot ANOVA yielded a significant main effect of treatment $\left(F_{(2,24)}=3.82 ; p<0.05\right)$ and its interaction with bins $\left(F_{(10,120)}=2.01 ; p<\right.$ 0.05). Post hoc comparisons confirmed that only the GD9-PolyI:C group differed significantly from offspring born to control dams $(p<0.05)$. This specific effect of GD9-PolyI:C treatment cannot be attributed to any changes in overall locomotor activity, because the total distance traveled in the open field never differed among groups (Fig. 1b). This represents a robust effect of mid- but not late-pregnancy immune activation (Shi et al., 2003; Meyer et al., 2005).

Second, the same animals were tested on discrimination reversal learning using a water T-maze. After learning to swim to one of the two stems of the T-maze to escape from the water, the animals were then required to learn to swim to the previously inescapable stem. A deficit in the second reversal phase, but not in the first acquisition phase, is indicative of perseverative behavior, as implicated in schizophrenia, autism, obsessive compulsive disorders, and addictive behavior (Ridley, 1994). Behavioral perseveration was only evident in the GD17-PolyI:C group but not the GD9-PolyI:C group. GD17-PolyI:C animals were uniquely impaired in the reversal phase (Fig. $2 a$, right). One-way ANOVA of the number of errors to criterion in the reversal phase yielded a clear main effect of treatment $\left(F_{(2,24)}=4.47 ; p<0.05\right)$ that is solely attributed to the perseverative performance of the GD17PolyI:C group compared with the control $(p<0.05)$ and GD9PolyI:C $(p<0.001)$ groups. No group difference was detected in the equivalent measure at the first acquisition phase (Fig. 2a, left), supporting the interpretation that the effect of GD17-PolyI:C treatment did not stem from a general learning deficit. A similar pattern of results is also obtained in the analyses of the daily performance in terms of choice accuracy (Fig. 2b). This illustrates that although the initial impact of reversal was comparable among the three groups, its influence on choice behavior remained in the GD17-PolyI:C group for another day while the other two groups were adapting to the reversed contingency. Analysis of the percentage of correct choice across the $5 \mathrm{~d}$ of reversal training yielded a significant main effect of treatment $\left(F_{(2,24)}=4.41 ; p<0.05\right)$ and its interaction with days $\left(F_{(8,96)}=\right.$ $3.91 ; p<0.001)$. Additional analyses on successive training days indicated that the GD17-PolyI:C group performed at a level significantly below that of the others $\left(F_{(2,24)}=6.29\right.$; $p<0.01$; post hoc, $p<0.01)$.

\section{Differential neuropathology in early adolescence after prenatal inflammation on middle and late gestation}

The dissociable behavioral outcomes between middle and late prenatal immune activation confirm our expectation that prena-
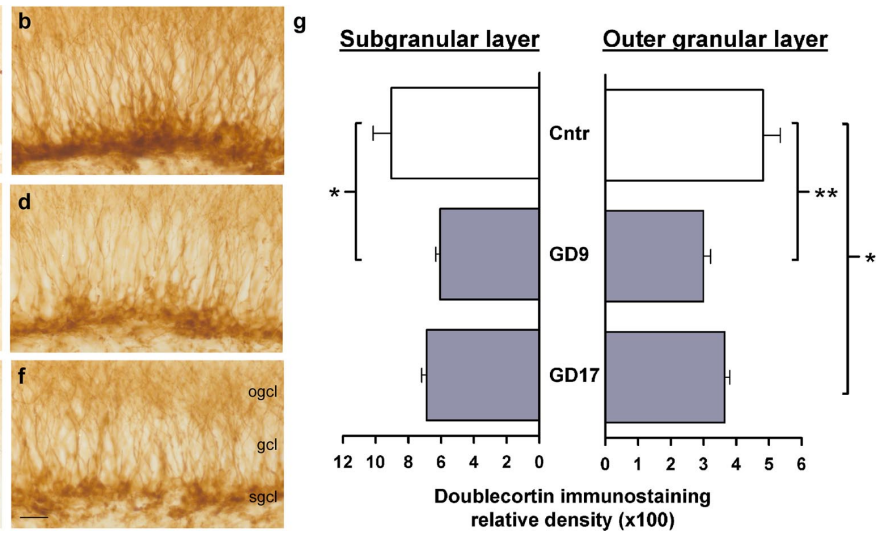

Figure 4. Reduction in postnatal neurogenesis in the dentate gyrus after prenatal immune challenge. $\boldsymbol{a}, \boldsymbol{c}, \boldsymbol{e}$, Photomicrographs of coronal brain sections of the hippocampal formation and dentate gyrus were taken from representative control offspring (a) and animals subjected to prenatal Polyl:C exposure on GD9 (c) or GD17 (e) and visualized with immunoperoxidase in $\boldsymbol{g}$ are mean \pm SEM. ${ }^{*} p<0.05$ and ${ }^{* *} p<0.01$, statistical significance based on Fisher's LSD post hoc analysis. Scale bars: $\boldsymbol{a}, \boldsymbol{c}$, $\boldsymbol{e}, 500 \mu \mathrm{m} ; \boldsymbol{b}, \boldsymbol{d}, \boldsymbol{f}, 50 \mu \mathrm{m}$. Cntr, Control; ogcl, outer granule cell layer; gcl, granule cell layer; sgcl, subgranular cell layer.

tal immune challenge at distinct prenatal times can differentially compromise the integrity of specific functional neural circuitries. To seek direct evidence for this hypothesis, we examined putative changes at the neuroanatomical level in brains collected from juvenile offspring (killed on P24) born to mothers treated with PolyI:C or vehicle on GD9 or GD17. Immunohistochemical analyses of three relevant markers are reported here.

First, we examined the expression of Reelin immunoreactivity because human postmortem studies have shown that reduced Reelin expression is associated with several neuropsychiatric disorders with a presumed neurodevelopmental origin (Fatemi, 2005). Prenatal PolyI:C exposure markedly reduced the number of hippocampal Reelin-positive cells, but this effect was more severe when the PolyI:C exposure took place at mid-pregnancy (Fig. 3). Stereological analyses in the hippocampus revealed a significant group difference in the number of Reelinimmunoreactive cells in stratum oriens of the dorsal CA1 subfield $\left(F_{(2,9)}=5.27 ; p<0.05\right)$. Subsequent post hoc comparisons verified that this stemmed from a significant reduction in the GD9PolyI:C group $(p<0.05)$ and a nonsignificant trend in the GD17-PolyI:C group, relative to control levels.

Although the number of Reelin-positive cells in the dentate gyrus was virtually unaffected by the prenatal treatment (means \pm SEM: control, 19,101.49 \pm 2142.8; GD9-PolyI:C, 15,295.9 \pm 2291.9; GD17-PolyI:C, 19,419.8 \pm 1546.5$)$, the Reelin immunoreactivity in the neuropil throughout the hippocampal formation appeared to be generally reduced in PolyI:C-treated subjects compared with controls (Fig. $3 a-c$ ). Considering that Reelin is involved in fetal and postnatal neural development by acting as a positioning and detachment signal for neurogenesis (Hack et al., 2002), the reduction in Reelin-positive cells in the CA area as well as the putative decrease in the amount of secreted protein in other subfields of the hippocampal formation suggest that these alterations might be accompanied by changes in the number of neuronal progenitor cells in the hippocampus. Indeed, using an antibody against DCX to visualize neuronal progenitor cells (Brown et al., 2003) in the dentate gyrus where postnatal/adult neurogenesis is most abundant, we further revealed that the levels of newly born neurons in the dorsal dentate gyrus were reduced in offspring born to PolyI:C-treated dams independent of prenatal treatment times (Fig. 4). These impressions were supported 


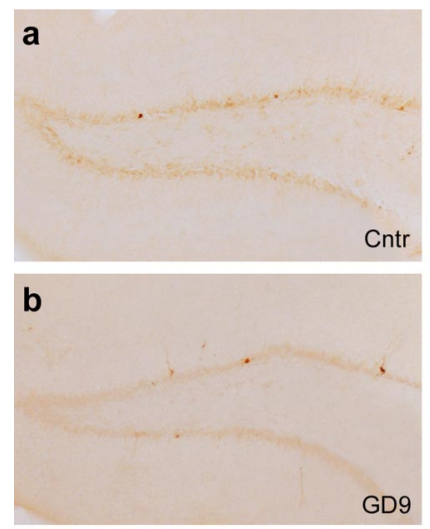

d
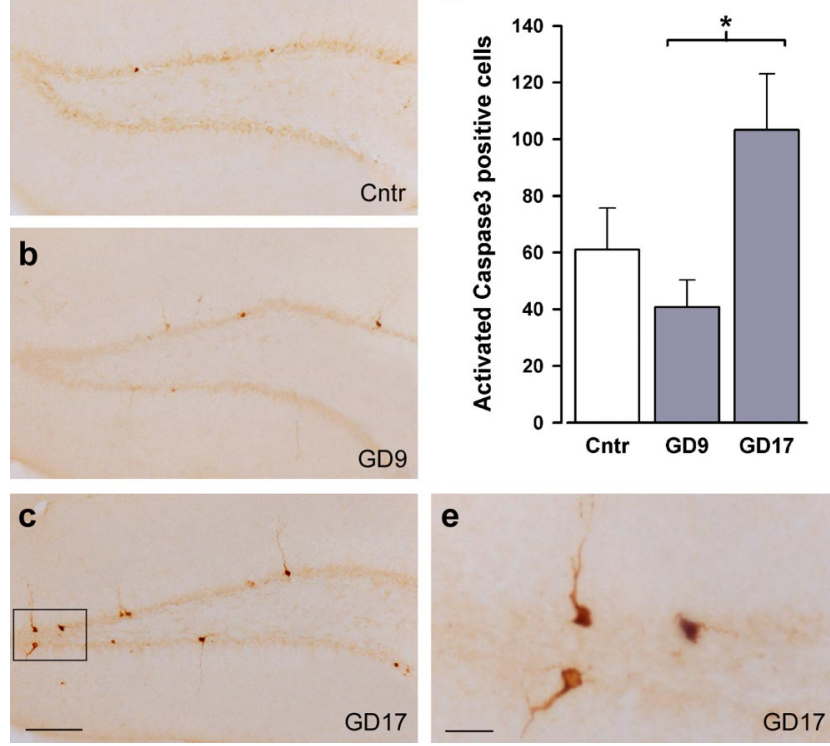

Figure 5. Selective enhancement of postnatal brain apoptosis after immune challenge on late but not middle gestation. $\boldsymbol{a}-\boldsymbol{c}, \boldsymbol{e}$, Distribution of activated caspase-3-immunoreactive cells in the dorsal dentate gyrus of representative control animals $(\boldsymbol{a})$ and offspring subjected to prenatal Polyl: $C$ exposure on GD9 (b) or GD17 ( $\boldsymbol{c}$; higher magnification in $\boldsymbol{e}$ ). $\boldsymbol{d}$, An increase in activated caspase-3-positive cells was observed exclusively in animals exposed to prenatal Polyl:C on GD17, whereas the same treatment on GD9 marginally reduced the number of caspase-3-positive cells compared with control brain tissue, leading to a significant difference between the two prenatal Polyl: C conditions. $n=4$ in each treatment group. The values in $\boldsymbol{d}$ are mean \pm SEM. ${ }^{*} p<0.05$, statistical significance based on Fisher's LSD post hoc analysis. Scale bars: $\boldsymbol{a}-\boldsymbol{c}, 500 \mu \mathrm{m} ; \boldsymbol{e}, 50 \mu \mathrm{m}$. Cntr, Control.

by the main effects of prenatal treatment attaining statistical significance in the ANOVAs conducted on the densitometric evaluation of DCX immunoreactivity in the subgranular $\left(F_{(2,9)}=5.11 ; p<0.05\right)$ and outer granule $\left(F_{(2,9)}=7.34 ; p<0.05\right)$ cell layers of the dentate gyrus. Subsequent post hoc analyses also showed that the reduction in DCX immunoreactivity in GD9-PolyI:C offspring was statistically significant in both the subgranular $(p<0.05)$ and outer granule $(p<0.01)$ cell layers compared with control offspring, whereas prenatal PolyI:C exposure on GD17 particularly reduced postnatal neurogenesis in the outer granule cell layer of the dentate gyrus $(p<$ 0.05 ) relative to prenatal control treatment (Fig. $4 g$ ).

The concomitant reduction in levels of Reelin and neurogenesis led us to speculate that they could be related to a putative increase in apoptotic cell loss in these juvenile offspring from PolyI:C-treated dams. Apoptotic cells were visualized by the immunostaining of activated caspase-3, a cysteine protease and a key degradative enzyme involved in apoptosis (Rami et al., 2003). Quantitative analysis of activated caspase-3- immunoreactive cells in the dentate gyrus granule cell layer indicated that increased apoptotic activity was exclusively found in offspring from the GD17-PolyI:C group (Fig. 5). This impression was supported by the main effect of prenatal treatment in the ANOVA of this measure $\left(F_{(2,9)}=4.37 ; p<0.05\right)$ and the subsequent post hoc comparisons revealing a statistically significant difference between offspring subjected to prenatal PolyI:C exposure on GD9 and GD17 $(p<0.05)$. The number of caspase-3immunoreactive cells was, if anything, reduced in the GD9-Poly group, albeit nonsignificantly so (Fig. $5 d$ ).

Consistent with our previous observations (M. Nyffeler, U. Meyer, B. K. Yee, J. Feldon, and I. Knuesel, unpublished results), prenatal PolyI:C treatment did not alter the overall morphology of the hippocampus, nor did it induce cell necrosis and reactive gliosis as confirmed by the qualitative evaluation of pyknotic cells or astrocytes (Fig. 6).

\section{Divergent cytokine reactions to maternal immune challenge at middle and late gestation by the maternal host and the fetus}

Next, we analyzed cytokine events taking place in the maternal serum and fetal brain at the time of maternal immune stimulation by contrasting the cytokine response induced by maternal PolyI:C treatment on GD9 and GD17 at two different sampling intervals ( 3 and $6 \mathrm{~h}$ after treatment). We focused here on IL- $1 \beta$, IL-6, IL-10, and TNF- $\alpha$ because of their crucial roles in modulating neuronal cell differentiation (Ling et al., 1998; Potter et al., 1999) and survival (Marx et al., 2001), as well as dendrite growth and complexity (Gilmore et al., 2004).

\section{Maternal serum protein levels}

As expected, the exposure to PolyI:C markedly increased the serum protein levels of the four cytokines in both gestation day conditions (Fig. 7), and these effects were generally more pronounced at $3 \mathrm{~h}$ after treatment (Fig. $7 a$ ) compared with the latter sampling interval at $6 \mathrm{~h}$ after treatment (Fig. $7 b$ ). These impressions were supported by the emergence of significant main effects of treatment (IL- $1 \beta: F_{(1,28)}=6.23, p<0.05$; IL-6: $F_{(1,28)}=40.97$, $p<0.001$; IL-10: $F_{(1,28)}=16.46, p<0.001$; TNF- $\alpha: F_{(1,28)}=5.06$, $p<0.05)$ and their interactions with sampling time (IL- $1 \beta$ : $F_{(1,28)}=6.23, p<0.05$; IL-6: $F_{(1,28)}=36.46, p<0.001$; TNF- $\alpha$ : $\left.F_{(1,28)}=4.78, p<0.05\right)$ in the $2 \times 2 \times 2$ (treatment $\times \mathrm{GD} \times$ sampling time) randomized block ANOVAs. The three-way interaction also attained statistical significance $\left(F_{(1,28)}=4.23 ; p<\right.$ $0.05)$ in the analysis of IL-10.

Subsequent post hoc comparisons were then conducted between the effects of PolyI:C treatment on GD9 (GD9-PolyI:C vs GD9saline) and on GD17 (GD17-PolyI:C vs GD17-saline), as well as between the efficacy of PolyI:C treatment at the two treatment days (GD9-PolyI:C vs GD17-PolyI:C) at each sampling interval (3 or $6 \mathrm{~h}$ after treatment). The analyses conducted at the earlier sampling interval showed that the PolyI:C-induced increases in maternal serum IL- $1 \beta$ and IL- 6 protein levels were mostly comparable between GD9 and GD17 dams compared with vehicle treatment at the corresponding gestation days, because significant differences were detected between the GD9-PolyI:C and GD9-saline conditions (IL-1 $\beta$, $p<0.01$; IL-6, $p<0.001)$ and between the GD17-PolyI:C and GD17-saline conditions (IL- $1 \beta, p<0.05$; IL-6, $p<0.01$ ). In addition, PolyI:C exposure significantly increased the serum IL-10 and TNF- $\alpha$ contents in both gestation day conditions relative to vehicle treatment (all $p<0.05$ ), yet these effects of the immune treatment were stronger in the GD9-PolyI:C condition compared with the GD17-PolyI:C condition at $3 \mathrm{~h}$ after treatment $(p<0.05$ for both cytokines) (Fig. 7a).

Indeed, the peak IL-10 response to PolyI:C in GD17 dams was detected at the later sampling time, leading to a significant difference between the GD17-PolyI:C and GD17-saline conditions $(p<0.01)$, as well as between the GD9-PolyI:C and GD17PolyI:C groups $(p<0.05)$ (Fig. $7 b)$ at $6 \mathrm{~h}$ after treatment. The serum IL-10 contents in PolyI:C-treated GD9 dams virtually returned to baseline levels at $6 \mathrm{~h}$ after treatment, but they were still significantly increased compared with saline-treated GD9 dams at this sampling interval $(p<0.05)$. Similarly, the serum IL-6 contents were still elevated in PolyI:C-treated GD9 and GD17 dams compared with the corresponding vehicle treatment at $6 \mathrm{~h}$ after treatment (GD9-PolyI:C vs GD9-saline, $p<0.05$; GD17- 
PolyI:C vs GD17-saline, $p<0.01$ ), but the PolyI:C-induced IL-6 response at this sampling interval was stronger in GD17 compared with GD9 dams $(p<0.05)$. Serum protein levels of IL- $1 \beta$ were below the detection limit $6 \mathrm{~h}$ after PolyI:C treatment, and no statistically significant differences in the TNF- $\alpha$ response were detected between the different treatment groups and gestation day conditions at the latter sampling time.

\section{Fetal brain protein levels}

Next, we compared the fetal brain cytokine responses to maternal PolyI:C or vehicle administration on middle or late gestation in terms of fetal brain protein levels sampled at 3 or $6 \mathrm{~h}$ after treatment. The average fetal brain cytokine levels obtained per progeny ( $n=3-5$ fetuses per progeny) in the two prenatal PolyI:C conditions (GD9 or GD17) were expressed as percentage of deviation from the mean levels obtained after saline treatment conducted on the same gestation day and at the corresponding post-treatment sampling interval ( 3 or $6 \mathrm{~h})$. At each sample interval, and for each cytokine protein, independent Student's $t$ tests were conducted between effects of PolyI:C treatment on GD9 (GD9-PolyI:C vs GD9-saline) and on GD17 (GD17PolyI:C vs GD17-saline), as well as between the efficacy of PolyI:C treatment at the two treatment days (GD9-PolyI:C vs GD17-PolyI:C).

Compared with vehicle treatment on the same day, PolyI:C exposure on GD9 reduced brain contents of IL-10 $(p<0.05)$ at $3 \mathrm{~h}$ after treatment, but the same treatment conducted on GD17 elevated the brain levels of this cytokine $(p<0.05)$ (Fig. $8 a)$, leading to a significant difference in the relative IL-10 protein contents between PolyI:Ctreated GD9 and GD17 fetal brain tissue $(p<0.001)$. The PolyI: $\mathrm{C}$-induced changes in fetal brain IL-10 protein at $3 \mathrm{~h}$ after treatment essentially returned to control levels $6 \mathrm{~h}$ after treatment (Fig. 8b). GD9-PolyI:C treatment was also unique in producing a massive elevation of IL-6 levels at the earlier sampling time relative to GD9-saline treatment $(p<0.01)$ and GD17-PolyI:C treatment $(p<0.001)$ (Fig. 8a). At $6 \mathrm{~h}$ after treatment, however, the PolyI:C-induced elevation of IL- 6 levels relative to vehicle treatment was primarily comparable between GD9-PolyI:C and GD17-PolyI:C groups (GD9, $p<0.01$; GD17, $p<0.05$ ) (Fig. 8b). In addition, late-pregnancy PolyI:C exposure increased the brain contents of IL- $1 \beta$ at $3 \mathrm{~h}$ after treatment relative to GD17-vehicle treatment $(p<0.05)$, but an opposite effect of the immune treatment was revealed in the GD9 condition $(p<0.01)$, leading to a significant difference between the two PolyI:C-exposed gestation day conditions in terms of the relative IL- $1 \beta$ protein contents $(p<0.001)$ (Fig. $8 a)$. At the later sampling interval, however, the fetal brain IL- $1 \beta$ levels were increased in GD9-PolyI:C fetuses compared with GD9-saline treatment $(p<0.01)$ and GD17-PolyI:C treatment $(p<0.001)$ (Fig. $8 b)$. No statistically significant differences in the relative TNF- $\alpha$ protein levels were detected between the PolyI:C and vehicle groups in both gestation day conditions, albeit a trend toward increased fetal brain TNF- $\alpha$ con-
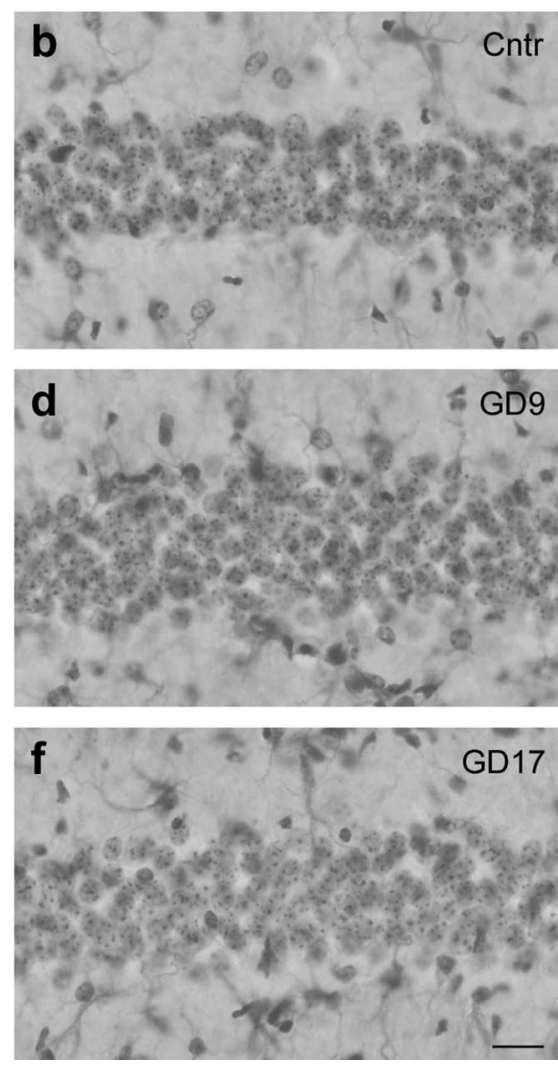

prenatal Polyl:C exposure. $\boldsymbol{a}, \boldsymbol{c}, \boldsymbol{e}$, Photomicrographs of corona Figure 6. Unaltered postnatal brain gross morphology after prenatal Polyl.C exposure. $a, c$,
brain sections of the dorsal hippocampal formation were taken from representative control animals $(\boldsymbol{a})$ and animals subjected to Polyl:C exposure on GD9 (c) or GD17 (e) and visualized with Nissl/GFAP double staining, which stains neuronal cell bodies or in the abundance of pyknotic neurons and astrocytes. Scale bars: $\boldsymbol{a}, \boldsymbol{c}, \boldsymbol{e}, 500 \mu \mathrm{m} ; \boldsymbol{b}, \boldsymbol{d}, \boldsymbol{f}, 50 \mu \mathrm{m}$.

tents was detected in PolyI:C-treated GD17 fetuses relative to GD17vehicle treatment at the later sampling time (Fig. $8 b$ ).

\section{Fetal brain gene expression levels}

Parallel gene expression analysis of fetal brain tissues also revealed clear differences in cytokine response induced by midversus late-gestational PolyI:C challenge as shown in Figure 9. As described above, the average fetal brain cytokine levels obtained per progeny ( $n=3-4$ fetuses per progeny) was submitted to statistical analyses. At each sample interval, and for each cytokine, independent Student's $t$ tests were conducted on the $\Delta$ threshold cycle $\left(\Delta \mathrm{C}_{\mathrm{T}}\right)$ values of the corresponding cytokines to assess the effects of PolyI:C treatment on GD9 (GD9-PolyI:C vs GD9saline) and on GD17 (GD17-PolyI:C vs GD17-saline) and to contrast the efficacy of PolyI:C treatment at the two treatment days (GD9-PolyI:C vs GD17-PolyI:C).

At $3 \mathrm{~h}$ after treatment, expression of IL- $1 \beta$ mRNA was suppressed to a similar degree by PolyI:C treatment on GD9 $(p<$ $0.05)$ and on GD17 $(p<0.05)$ compared with vehicle treatment (Fig. 9a). No effect was observed in IL-6 and IL-10 mRNA levels at this sampling time. A weak differential response was seen in TNF- $\alpha$ gene expression, with a significant reduction only detected in the GD17-PolyI:C group $(p<0.05)$ relative to GD17 vehicle treatment (Fig. 9a). No differences in the relative cytokine gene expression were detected between PolyI:C exposure on GD9 and GD17.

The clearest evidence for a critical dependency on the time of gestational PolyI:C challenge are derived at $6 \mathrm{~h}$ after treatment (Fig. $9 b)$. GD17-PolyI:C treatment led to a significant elevation in the 
a
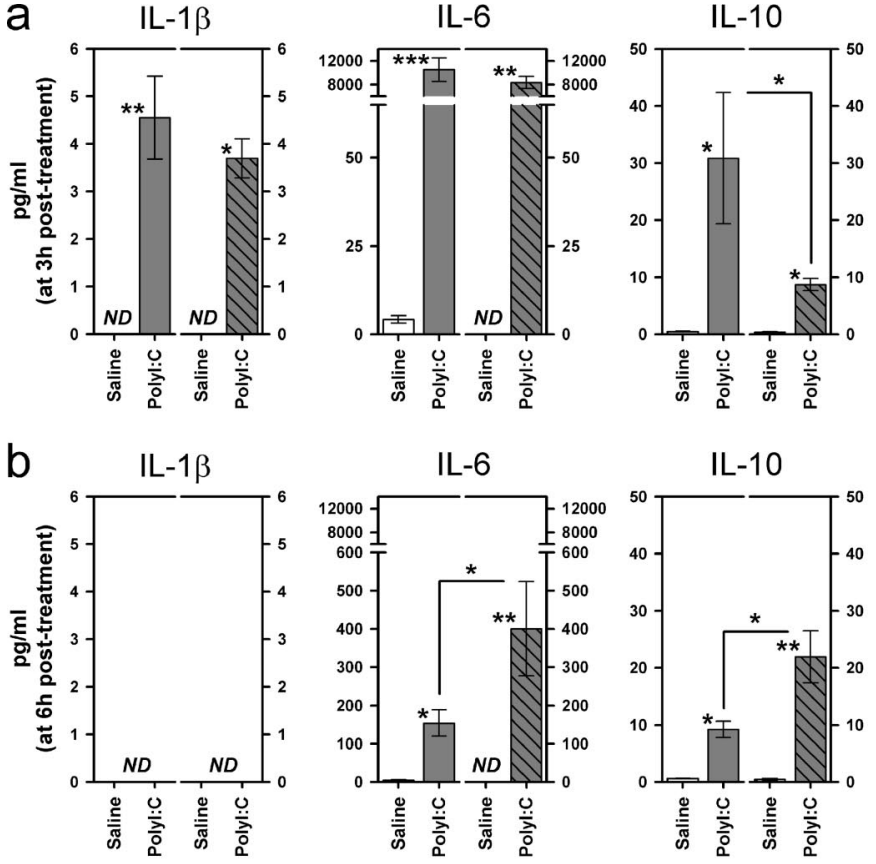

NIV Saline treatment on GD17

Saline treatment on GD9

Polyl:C treatment on GD9

MIV Polyl:C treatment on GD17

Figure 7. Comparison of the maternal serum cytokine responses after immune challenge in middle and late gestation. The maternal administration of Polyl:C ( $5 \mathrm{mg} / \mathrm{kg}$, i.v.) led to a marked increase in the serum protein levels of IL- $1 \beta$, IL-6, IL-10, and TNF- $\alpha$ in both gestation day conditions relative to vehicle (saline) treatment, and these effects were generally more pronounced at $3 \mathrm{~h}$ after treatment $(\boldsymbol{a})$ compared with the later sampling time at $6 \mathrm{~h}$ after treatment $(\boldsymbol{b})$. However, whereas the Polyl:C-induced IL-10 and TNF- $\alpha$ responses were stronger in GD9 compared with GD17 dams at the earlier sampling interval, higher serum protein levels of IL- 6 and IL-10 were detected in Polyl:C-exposed GD17 dams compared with the Polyl:C-treated GD9 group at $6 \mathrm{~h}$ after treatment. The number of pregnant dams in each group was 4, except in the following three groups: GD9-Polyl:C/3 h, $n=6$; GD17-Polyl:C/3h, $n=5 ; G D 17-P o l y l: C / 6 h, n=5$. The levels of IL- $1 \beta$ in vehicle-treated animals were always below the detection limit (ND), regardless of the gestation day condition, and so were the IL-6 levels in the vehicle-treated GD17 group at both sampling intervals. All values are mean \pm SEM. ${ }^{*} p<0.05,{ }^{* *} p<0.01$, and ${ }^{* * *} p<0.001$, statistical significance based on Fisher's LSD post hoc comparisons between the effects of Polyl:C treatment on GD9 (GD9-Polyl:C vs GD9-saline) and on GD17 (GD17-Polyl:C vs GD17-saline), as well as between the efficacy of Polyl:C treatment at the two treatment days (GD9-Polyl:C vs GD17-Polyl:C) at each sampling interval (3 or $6 \mathrm{~h}$ after treatment).

gene expression levels of IL-6 ( $p<0.01)$, IL-10 $(p<0.05)$, and TNF- $\alpha(p<0.01)$ relative to GD17-saline treatment. In contrast, GD9-PolyI:C treatment suppressed IL-10 gene expression $(p<$ 0.01 ) compared with GD9 vehicle treatment and exerted little influence on the gene expression levels of the other three cytokines (Fig. $9 b$ ). This led to the emergence of significant differences between the PolyI:C-exposed GD9 and GD17 groups in the relative gene expression levels of IL- $1 \beta(p<0.05)$, IL-6 $(p<0.01)$, IL-10 $(p<0.001)$, and TNF- $\alpha(p<0.001)$.

\section{Discussion}

The present study shows that the time of maternal immune challenge critically determines the patterns of behavioral abnormalities displayed by the offspring at adult age. PolyI:C challenge on GD9 suppressed spatial exploration, whereas the same treatment conducted on GD17 led to perseverative behavior. In each case, the deficit was not seen in the other treatment condition, thus demonstrating a cross-double dissociation of the long-term behavioral effects associated with maternal immune activation in mid and late gestation. This extends the single dissociation we observed previously, in which GD9-PolyI:C but not GD17PolyI:C treatment impaired the latent inhibition effect of associative learning (Meyer et al., 2006). Hence, prenatal immune activation can lead to the long-term development of specific psycho- pathological traits (Shi et al., 2003; Zuckerman et al., 2003; Meyer et al., 2005, 2006), but the specificity of this vulnerability depends on the gestational time windows at which the maternal host is infected.

Here, we have extended the divergence in adult psychopathology between midand late-gestational immune activation to the postnatal emergence of brain neuropathology. The reduction in hippocampal Reelin expression was most pronounced after immune challenge at mid-pregnancy, but the increase in apoptosis was observed exclusively after late-gestational immune activation. Both of these histopathological markers have been implicated in the disease process of autism and schizophrenia (Araghi-Niknam and Fatemi, 2003; Fatemi, 2005; Jarskog et al., 2005). However, the causes of these structural alterations are not known. When turned to the fetal developmental stage, we demonstrated that cytokine-related inflammatory processes in prenatal life are potential causal agents of postnatal brain histopathology and that the precise outcomes, similar to the adult psychopathology, depend critically on the time of their activation. It is important to emphasize that we did not observe any sign of neurodegeneration that is typified by the presence of necrotic cell loss and reactive gliosis. The absence of gross brain anatomical alterations agrees well with the hypothesis that neurodevelopmental disturbance rather than neurodegeneration is central to the etiopathogenesis and disease process of schizophrenia and autism (Weinberger, 1987; Rodier and Hyman, 1998; Arndt et al., 2005; Miller et al., 2005; Rapoport et al., 2005).

Reduced Reelin expression in the cortex and hippocampus has been reported in neonatal offspring from dams having been infected with influenza virus at mid-pregnancy (Fatemi et al., 1999). Our data confirm that maternal inflammation in the absence of a specific viral pathogen is similarly capable to suppress Reelin expression in the postnatal brain. This further supports the hypothesis that the critical casual link in the association between maternal infection during pregnancy and increased risk of mental disorders in the offspring is the maternal immunological response to infection (Gilmore and Jarskog, 1997; Shi et al., 2005).

Among the various immunological events that can be triggered by infectious agents, the cytokine-associated inflammatory response may be of particular relevance. Indeed, elevations of inflammatory cytokines in the maternal host and eventually in the fetal environment may represent one of the key events in triggering or precipitating neurodevelopmental dysfunctions (Gilmore and Jarskog, 1997; Buka et al., 2001; Brown et al., 2004a). Here, we provide the first evidence that maternal inflammation induced at different times with divergent pathological outcomes in brain and behavior are also accompanied by dissociable cytokine responses in the fetal brain shortly after the induction event. This completes the neurobiological 
differentiation between mid- and lategestational immune challenge from adult behavior, postnatal neuroanatomy to fetal immunology in utero.

Importantly, we reveal that the fetal brain may directly contribute to the specific changes in cytokine protein levels after maternal infection at late but not at mid gestation. This distinction cannot be attributed to a differential efficacy of the immune treatment at the two gestation times to induce significant cytokine responses in the host's peripheral immune system, because the administration of PolyI:C markedly elevated maternal serum cytokine levels in both middle and late pregnancy (Fig. 7). Moreover, because the pronounced elevation of proinflammatory cytokine proteins in fetal brain tissue after immune challenge in mid gestation was not accompanied by parallel increases in the mRNA expression levels of the corresponding genes, the cytokines protein elevation cannot be readily accounted by an endogenous increase in cytokine production. Rather, the excess cytokine proteins may be of maternal origin (Zaretsky et al., 2004) and/or of units of the maternal-fetal interface, including the placenta (Bowen et al., 2000). However, the present data do not exclude the possibility that alternative mechanisms such as variations in posttranscriptional processing (Clark, 2000), which are relatively poorly characterized in the fetal immune system, could also have contributed to the dissociation of cytokine protein and gene expression levels in PolyI:C-exposed GD9 fetal brains. In contrast, immune challenge conducted in late gestation induced elevations of both pro- and anti-inflammatory cytokine proteins, with concomitant increases in the relative expression of the corresponding genes. This latter finding is in full agreement with a recent study using bacterial endotoxin as an immunestimulating agent in pregnant rats at late gestation (Cai et al., 2000) and readily suggests that the observed increase in specific cytokines after maternal immune activation on late gestation might, at least in part, be attributable to endogenous production by the fetal brain.

The possibility that cytokine-related events in utero may precipitate postnatal structural alternations in the brain is highlighted by the specific concordant as well as discordant effects emerged from the comparisons between the cytokine response patterns after mid- and late-gestational exposure to PolyI:C. These may be further correlated with the histopatholology observed in the postnatal brain.

Late-gestational immune challenge uniquely activated both IL-10 and TNF- $\alpha$ response in the fetal brain and is associated with increased postnatal apoptosis. TNF- $\alpha$ is a potent inducer of apoptotic processes in a variety of neuronal and non-neuronal cells in acute preparations (Tracey and Cerami, 1993). IL-10, on the other hand, can impede or even prevent the progression of apoptosis (e.g., via inhibition of TNF- $\alpha$ production and activity)
(Bachis et al., 2001). Hence, when the protein levels of IL-10 were elevated at $3 \mathrm{~h}$ after treatment, TNF- $\alpha$ gene expression was concomitantly reduced. Yet, TNF- $\alpha$ gene expression doubled, and its protein levels rose by $50 \%$ by $6 \mathrm{~h}$ after treatment, when IL-10 levels had returned to the baseline level. Although the persistence of the increase in TNF- $\alpha$ gene expression was not precisely determined here, viral infection on $\mathrm{P} 1$ has been reported to induce a sustained elevation of TNF- $\alpha$ levels for 4 weeks (Hornig et al., 1999).

A previous study reported that the prenatal administration of PolyI:C on mid to late gestation in rats reduced the protein levels of TNF- $\alpha$ in the neonatal brain (Gilmore et al., 2005). This recent finding seems to disagree with the suggestion that increased postnatal brain apoptosis in GD17-PolyI:C offspring may be directly associated with the efficacy of late-gestational PolyI:C exposure to induce significant TNF- $\alpha$ responses in the fetal brain. However, the efficacy of mid- to late-gestational immune challenge in rats to reduce the TNF- $\alpha$ protein contents in the brains of neonates was not associated with a similar reduction in TNF- $\alpha$ at the fetal brain level (Gilmore et al., 2005). This indicates that the acute immunological consequences of the maternal treatment on the fetal system may differ considerably between rats and mice. Conversely, the efficacy of maternal PolyI:C exposure to increase fetal brain TNF- $\alpha$ levels may also be critically determined by the exact maternal dosage and/or administration 

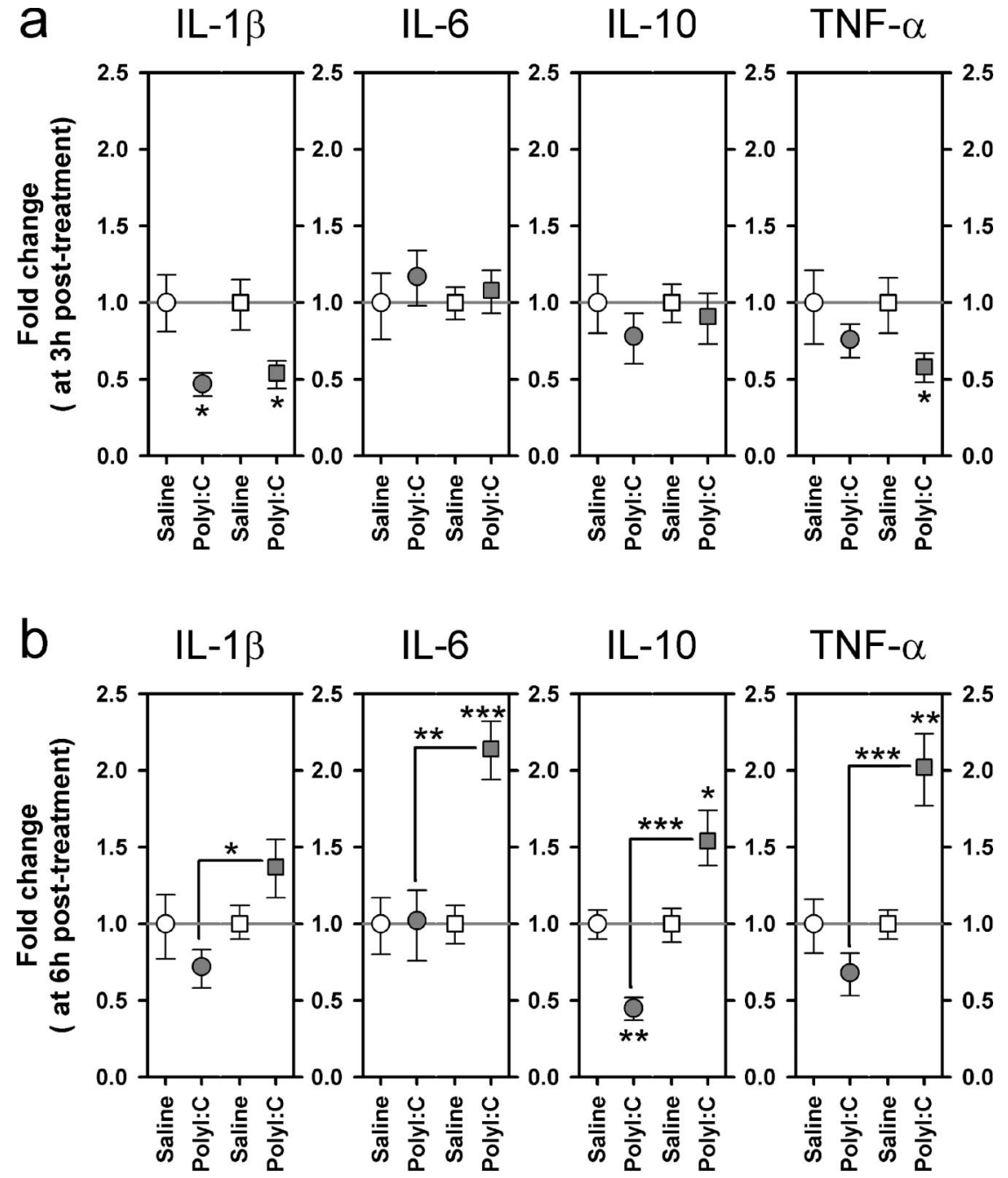

Saline treatment on GD9 Polyl:C treatment on GD9
Saline treatment on GD17 Polyl:C treatment on GD17
Figure 9. Comparison of the fetal brain cytokine responses after immune challenge in middle and late gestation in terms of relative gene expression levels. The graphs illustrate the fold changes in the relative cytokine gene expression as calculated using the $2^{-\Delta \Delta \mathrm{C}}$ method; the bars correspond to the minus and plus range of the fold changes. At each sample interval ( 3 and $6 \mathrm{~h}$ ), the average fetal brain cytokine levels obtained per progeny ( $n=3-4$ fetuses per progeny) was submitted to statistical analyses. $\boldsymbol{a}$, At $3 \mathrm{~h}$ after treatment, the relative gene expression levels of $I L-1 \beta$ were reduced in the fetal brains as a result of maternal Polyl:C exposure in both gestation day conditions, whereas a similar reduction in TNF- $\alpha$ expression was only observed in the brains of fetuses derived from immune-challenged GD17 dams. The relative expression levels of IL-6 and IL-10 genes were unaffected by the maternal treatment at this sampling time. $\boldsymbol{b}, \mathrm{At}$ $6 \mathrm{~h}$ after treatment, maternal immune stimulation on GD17 significantly increased the relative gene expression levels of IL-6, LL-10, and TNF- $\alpha$, whereas the same treatment conducted on GD9 exerted an opposite effect on the relative IL-10 gene expression levels. The number of pregnant dams in each group was 4, except in the following three groups: GD9-Polyl: $/ / 3 \mathrm{~h}, n=6 ; \mathrm{GD} 17-\mathrm{Polyl}: \mathrm{C} / 3 \mathrm{~h}, n=5$; GD17-Polyl:C/6 h, $n=5 .{ }^{*} p<0.05$, ${ }^{* *} p<0.01$, and ${ }^{* *} p<0.001$, statistical significance based on independent Student's $t$ tests between the effects of Polyl:C treatment on GD9 (GD9-Polyl:C vs GD9-saline) and on GD17 (GD17-Polyl:C vs GD17-saline), as well as between the efficacy of Polyl: C treatment at the two treatment days (GD9-Polyl:C vs GD17-Polyl:C) at each sample interval ( 3 or 6 h).

between high levels of hippocampal proinflammatory cytokines (including IL-6) in the hippocampus and reduced neurogenesis in the dentate gyrus in adult animals (Vallieres et al., 2002; Monje et al., 2003). Our data thus also highlight the possibility that an acute inflammation of the fetal brain is capable to undermine an important form of structural plasticity during postnatal neurodevelopment.

Hence, there is clear evidence to suggest that the time of maternal viral-like infection determines the specificity of the acute cytokine events in the fetus, the developmental disturbances in the postnatal brain, and the psychopathological profile in adulthood. One implication is that the precise timing may underlie the relationship between the differing neurodevelopmental vulnerability associated with in utero infection and the etiopathogenesis of mental disorders with a presumed developmental origin. This may also determine whether the eventual psychological profile falls into one or another subtype category, given that different subtypes of a psychiatric disease are likely to be associated with distinct neurodevelopmental failures (Murray et al., 1992; Rodier and Hyman, 1998; Sporn et al., 2004).

\section{References}

Araghi-Niknam M, Fatemi SH (2003) Levels of Bcl-2 and P53 are altered in superior frontal and cerebellar cortices of autistic subjects. Cell Mol Neurobiol 23:945-952.

Arndt TL, Stodgell CJ, Rodier PM (2005) The teratology of autism. Int $\mathrm{J}$ Dev Neurosci 23:189-199.

Bachis A, Colangelo AM, Vicini S, Doe PP, De Bernardi MA, Brooker G, Mocchetti I (2001) Interleukin-10 prevents glutamatemediated cerebellar granule cell death by blocking caspase-3-like activity. J Neurosci 21:3104-3112.

Bowen JM, Chamley L, Keelan JA, Mitchell MD (2000) Cytokines of the placenta and extraplacental membranes: roles and regulation during human pregnancy and parturition. Placenta 23:257-273.

Brown AS, Hooton J, Schaefer CA, Zhang H, Petkova E, Babulas V, Perrin M, Gorman JM, Susser ES (2004a) Elevated maternal interleukin-8 levels and risk of schizophrenia in adult offspring. Am J Psychiatry 161:889-895.

Brown AS, Begg MD, Gravenstein S, Schaefer CA, routes. A direct comparison of cytokine events taking place in the fetal environment after the maternal exposure to inflammatory agents across different doses would therefore be of great value to further consolidate the putative causal relationship between enhanced TNF- $\alpha$ activity during fetal neurodevelopment and increased incidence of postnatal brain apoptosis.

Here, we also demonstrate that both early and late gestational PolyI:C treatment are equivalently effective in suppressing postnatal neurogenesis in the hippocampus. In terms of the acute effects of brain cytokines, PolyI:C treatment on either GD9 or GD17 increased IL-6 protein levels in the fetal brain. This parallels reports of a link
Wyatt RJ, Bresnahan M, Babulas VP, Susser ES (2004b) Serologic evidence of prenatal influenza in the etiology of schizophrenia. Arch Gen Psychiatry 61:774-780.

Brown JP, Couillard-Despres S, Cooper-Kuhn CM, Winkler J, Aigner L, Kuhn HG (2003) Transient expression of doublecortin during adult neurogenesis. J Comp Neurol 467:1-10. (2001) Maternal cytokine levels during pregnancy and adult psychosis. Brain Behav Immunol 15:411-420.

Cai Z, Pan ZL, Pang Y, Evans OB, Rhodes PG (2000) Cytokine induction in fetal rat brains and brain injury in neonatal rats after maternal lipopolysaccharide administration. Pediatr Res 47:64-72.
Buka SL, Tsuang MT, Torrey EF, Klebanoff MA, Wagner RL, Yolken RH 
Clark A (2000) Post-transcriptional regulation of pro-inflammatory gene expression. Arthritis Res 2:172-174.

Curfs JH, Meis JF, Hoogkamp-Korstanje JA (1997) A primer on cytokines: sources, receptors, effects, and inducers. Clin Microbiol Rev 10:742-780.

Dammann O, Leviton A (1997) Maternal intrauterine infection, cytokines, and brain damage in the preterm newborn. Pediatr Res 42:1-8.

Dammann O, Leviton A (2000) Role of the fetus in perinatal infection and neonatal brain damage. Curr Opin Pediatr 12:99-104.

Fatemi SH (2005) Reelin glycoprotein: structure, biology and roles in health and disease. Mol Psychiatry 10:251-257.

Fatemi SH, Emamian ES, Kist D, Sidwell RW, Nakajima K, Akhter P, Shier A, Sheikh S, Bailey K (1999) Defective corticogenesis and reduction in Reelin immunoreactivity in cortex and hippocampus of prenatally infected neonatal mice. Mol Psychiatry 4:145-154.

Fortier ME, Kent S, Ashdown H, Poole S, Boksa P, Luheshi GN (2004) The viral mimic, polyinosinic:polycytidylic acid, induces fever in rats via an interleukin-1-dependent mechanism. Am J Physiol Regul Integr Comp Physiol 287:R759-R766.

Gilmore JH, Jarskog LF (1997) Exposure to infection and brain development: cytokines in the pathogenesis of schizophrenia. Schizophr Res 24:365-367.

Gilmore JH, Jarskog LF, Vadlamudi S, Lauder JM (2004) Prenatal infection and risk for schizophrenia: IL-1beta, IL-6, and TNFalpha inhibit cortical neuron dendrite development. Neuropsychopharmacology 29:1221-1229.

Gilmore JH, Jarskog LF, Vadlamudi S (2005) Maternal poly I:C exposure during pregnancy regulates TNF alpha, BDNF, and NGF expression in neonatal brain and the maternal-fetal unit of the rat. J Neuroimmunol 159:106-112.

Gundersen HJ, Bagger P, Bendtsen TF, Evans SM, Korbo L, Marcussen N, Moller A, Nielsen K, Nyengaard JR, Pakkenberg B, Sørensen FB, Vesterby A, West MJ (1988) The new stereological tools: disector, fractionator, nucleator and point sampled intercepts and their use in pathological research and diagnosis. APMIS 96:857-881.

Hack I, Bancila M, Loulier K, Carroll P, Cremer H (2002) Reelin is a detachment signal in tangential chain-migration during postnatal neurogenesis. Nat Neurosci 5:939-945.

Hornig M, Weissenbock H, Horscroft N, Lipkin WI (1999) An infectionbased model of neurodevelopmental damage. Proc Natl Acad Sci USA 96:12102-12107.

Howard CV, Reed MG (2005) Unbiased stereology. New York: Garland Science/BIOS Scientific Publishers.

Jarskog LF, Glantz LA, Gilmore JH, Lieberman JA (2005) Apoptotic mechanisms in the pathophysiology of schizophrenia. Prog Neuropsychopharmacol Biol Psychiatry 29:846-858.

Kaufman MH (2003) The atlas of mouse development. London: Academic.

Ling ZD, Potter ED, Lipton JW, Carvey PM (1998) Differentiation of mesencephalic progenitor cells into dopaminergic neurons by cytokines. Exp Neurol 149:411-423.

Livak KJ, Schmittgen TD (2001) Analysis of relative gene expression data using real-time quantitative PCR and the 2(-Delta Delta $\mathrm{C}(\mathrm{T}))$ method. Methods 25:402-408.

Marx CE, Jarskog LF, Lauder JM, Lieberman JA, Gilmore JH (2001) Cytokine effects on cortical neuron MAP-2 immunoreactivity: implications for schizophrenia. Biol Psychiatry 50:743-749.

Mednick SA, Machon RA, Huttunen MO, Bonett D (1988) Adult schizophrenia following prenatal exposure to an influenza epidemic. Arch Gen Psychiatry 45:189-192.

Meyer U, Feldon J, Schedlowski M, Yee BK (2005) Towards an immunoprecipitated neurodevelopmental animal model of schizophrenia. Neurosci Biobehav Rev 29:913-947.

Meyer U, Feldon J, Schedlowski M, Yee BK (2006) Immunological stress at the maternal-foetal interface: a link between neurodevelopment and adult psychopathology. Brain Behav Immunol, in press.
Miller MT, Stromland K, Ventura L, Johansson M, Bandim JM, Gillberg C (2005) Autism associated with conditions characterized by developmental errors in early embryogenesis: a mini review. Int J Dev Neurosci 23:201-219.

Monje ML, Toda H, Palmer TD (2003) Inflammatory blockade restores adult hippocampal neurogenesis. Science 302:1760-1765.

Murray RM, O'Callaghan E, Castle DJ, Lewis SW (1992) A neurodevelopmental approach to the classification of schizophrenia. Schizophr Bull 18:319-332.

O'Callaghan E, Sham PC, Takei N, Murray G, Glover G, Hare EH, Murray RM (1994) The relationship of schizophrenic births to 16 infectious diseases. Br J Psychiatry 165:353-356.

Pearce BD (2001) Schizophrenia and viral infection during neurodevelopment: a focus on mechanisms. Mol Psychiatry 6:634-646.

Potter ED, Ling ZD, Carvey PM (1999) Cytokine-induced conversion of mesencephalic-derived progenitor cells into dopamine neurons. Cell Tissue Res 296:235-246.

Rami A, Jansen S, Giesser I, Winckler J (2003) Post-ischemic activation of caspase- 3 in the rat hippocampus: evidence of an axonal and dendritic localisation. Neurochem Int 43:211-223.

Rantakallio P, von Wendt L (1985) Risk factors for mental retardation. Arch Dis Child 60:946-952.

Rapoport JL, Addington AM, Frangou S, Psych MR (2005) The neurodevelopmental model of schizophrenia: update 2005. Mol Psychiatry 10:434-449.

Revello MG, Gerna G (2004) Pathogenesis and prenatal diagnosis of human cytomegalovirus infection. J ClinVirol 29:71-83.

Ridley RM (1994) The psychology of perserverative and stereotyped behaviour. Prog Neurobiol 44:221-231.

Rodier PM, Hyman SL (1998) Early environmental factors in autism. Men Retard Dev Disabil Res Rev 4:121-128.

Sargent IL (1993) Maternal and fetal immune responses during pregnancy. Exp Clin Immunogenet 10:85-102.

Shi L, Fatemi SH, Sidwell RW, Patterson PH (2003) Maternal influenza infection causes marked behavioral and pharmacological changes in the offspring. J Neurosci 23:297-302.

Shi L, Tu N, Patterson PH (2005) Maternal influenza infection is likely to alter fetal brain development indirectly: the virus is not detected in the fetus. Int J Dev Neurosci 23:299-305.

Sporn AL, Addington AM, Gogtay N, Ordonez AE, Gornick M, Clasen L, Greenstein D, Tossell JW, Gochman P, Lenane M, Sharp WS, Straub RE, Rapoport JL (2004) Pervasive developmental disorder and childhood-onset schizophrenia: comorbid disorder or a phenotypic variant of a very early onset illness? Biol Psychiatry 55:989-994.

Tracey KJ, Cerami A (1993) Tumor necrosis factor, other cytokines and disease. Annu Rev Cell Biol 9:317-343.

Vallieres L, Campbell IL, Gage FH, Sawchenko PE (2002) Reduced hippocampal neurogenesis in adult transgenic mice with chronic astrocytic production of interleukin-6. J Neurosci 22:486-492.

Vignali DA (2000) Multiplexed particle-based flow cytometric assays. J Immunol Methods 243:243-255.

Weinberger DR (1987) Implications of normal brain development for the pathogenesis of schizophrenia. Arch Gen Psychiatry 44:660-669.

Wu YW (2002) Systematic review of chorioamnionitis and cerebral palsy. Ment Retard Dev Disabil Res Rev 8:25-29.

Wu YW, Colford Jr JM (2000) Chorioamnionitis as a risk factor for cerebral palsy: a meta-analysis. JAMA 284:1417-1424.

Zaretsky MV, Alexander JM, Byrd W, Bawdon RE (2004) Transfer of inflammatory cytokines across the placenta. Obstet Gynecol 103:546-550.

Zuckerman L, Rehavi M, Nachman R, Weiner I (2003) Immune activation during pregnancy in rats leads to a postpubertal emergence of disrupted latent inhibition, dopaminergic hyperfunction, and altered limbic morphology in the offspring: a novel neurodevelopmental model of schizophrenia. Neuropsychopharmacology 28:1778-1789. 